\title{
Gamification of Immersive Meditation Practice in Virtual Reality
}

\author{
Matthew Moroz \\ Department of Psychology \\ University of Nevada, Reno \\ Nevada, USA \\ mmoroz@nevada.unr.edu
}

\author{
Bianca Calagiu \\ Fairhaven College of Interdisciplinary Studies \\ Western Washington University \\ Washington, USA \\ calagib@wwu.edu
}

\begin{abstract}
Benefits arising from meditation practice gamification are not overtly obvious. Desires to achieve and progress to higher levels, which are common to gaming, seem diametrically opposed to the ethos underlying traditional meditation practice. We propose, however, that a motivation to gain greater wellbeing and enlightenment via mindfulness meditation practice shares more with the motivation to progress through a game than is initially apparent. We begin by explaining how gamification techniques may be employed in meditation practice with a focus on mitigating the five hindrances to successful practice as described in the Theravada tradition. We then highlight the utility of employing virtual reality as a medium for such simulations. We discuss the potential for beneficial therapeutic applications in patients with mental health disorders and prison populations. We conclude by summarising our position and urging increased attention in this increasingly relevant area of research.
\end{abstract}

Keywords-Virtual reality; meditation; Vipassana; mental health

\section{INTRODUCTION}

We see many signs of increased popularity and awareness of mindfulness and other types of meditation $[1,2]$. Many videos and applications are now available to users providing advice and guidance. Meditation practice gamification is a potentially divisive concept. One might argue that a subset of the factors which make gamification so useful are exactly those with which one attempts to suppress using meditation. Games can increase a user's distractedness, provoke desire for completion or leveling up, and may generally add to a feeling of unsatisfactoriness [3, 4].

Superficially there are few similarities to the gamification of other 'wellness' applications such as Strava [5] or Zwift [6]. However, we draw much hope from the success of such applications and look to employ the most relevant aspects from each. The type of meditation practice we shall describe may seem unpalatable to traditional practitioners. However, the shared themes with traditional gaming, and potential benefits for users, make pursuing this genre of gaming a worthwhile endeavor.
Much research into the benefits of Vipassana (mindfulness), and other types of meditation practice, has taken place over in recent years [7,8]. Studies demonstrate beneficial results for practitioners in general, and in more specific groups such as those suffering various psychiatric disorder [9].

Of Buddhist traditions still practiced, Theravada has the longest history. Originally developed in Sri Lanka, the doctrine remains dominant in it's birthplace and remains similarly relevant in Burma, Laos, Cambodia and Thailand [10]. While the original form is considered a very conservative branch of Buddhism, modern versions stemming from Theravada continue to propagate including Vipassana [11]. Theravada tradition identifies five obstacles to effective, regular mindfulness meditation practice and the intended stages of concentration [12]. They come in the shape of sensory desire, ill will, sloth/torpor, restlessness/remorse, and doubt $[13,14]$. These translate into many practitioners, especially those new to practice, quickly becoming bored, frustrated, and disillusioned. We describe how gamification offers antidotes to each of these obstacles.

\section{Gamifying Meditation Practice}

When considering the gamification of meditation practice we take much from the successful implementation and popularity of running/cycling applications Strava and Zwift. Each began as fun, interactive, applications initially used by cyclists before expanding to include the runners. We could argue that many cyclists and runners shall naturally gravitate towards tech and gadgets which complement their athletic progress due to a heightened competitive nature. The techniques employed by these applications to entertain, inspire, and motivate, are however, directly transferable.

We may describe Strava as 'Facebook for runners and cyclists'. Users post photographs taken during their activities. Fellow users offer 'kudos' rather than simply 'liking' other's activities. Monthly challenges help to galvanize users to push themselves. The communitystyle platform encourages support, interactivity, and inclusion for all. Whatever a user's level of athletic ability they are helped to feel a real sense of achievement. 
Zwift differs to Strava in that it focuses on creating an environment in which those restricted to indoor cycle or treadmill may escape the monotony of their surroundings. Users may choose to virtually ride the roads of London, Richmond (VA), or the fictional land of Watopia. Virtual versions of London and Richmond closely mimic the real-world. Those unfamiliar with the cities enjoy something of a touristic visit. Those familiar may enjoy the nostalgia afforded by the detailed mock-ups. All environments allow users to test themselves against others inhabiting the virtual roads at the same time. These can come in the form of other users unknown to a particular individual, or friends from distant corners of the globe who've arranged to meet up and ride together online.

Taking inspiration from each platform and implementing the most relevant and beneficial aspects for mediation practice shall increase both motivation and engagement. By offering a Strava-like community platform for meditation practice we reduce the potential for boredom and loneliness by vastly increasing social interaction and community support [15]. Users may passively engage in the community or more actively arrange group practice sessions as is possible with Zwift. Continued participation is motivated by such camaraderie alongside more competitive motivations to gain in-app rewards such as badges earned by completing self or app prescribed challenges [16]. Successful competition may by accompanied by bonus environments and other relevant content to further encourage continued engagement. We may readily imagine fellow meditation practitioners offering 'namaste' is in recognition rather than a 'Facebook like' $\mathrm{B}$ or 'Strava kudos' 1 .

We consider each of the five hindrances and advance gamification techniques as mitigators. We may define each candidate technique as a 'gamified hindrance antidote'.

\section{A. Sensory Desire}

The human desire for visual, auditory, olfactory, gustatory and somatosensory stimulation is innate [17]. Meditation attempts to quieten these desires and focus the attention. While absolute focus is impossible we are encouraged to view distracting thoughts as they enter consciousness, and send them on their way. Sensory desires have the potential to define our behavior in the moment and monopolize our thoughts [18].

In Zwift, Watopia is designed as a cyclist's paradise. We may offer similarly tailored environments to specifically encourage users by mitigating the problem of sensory desire. A new user may find solace from boredom by undertaking their meditation on a deserted beach which offers the perfect balance of sensory stimulation without crossing over to distraction. As a user progresses in their practice they may work through different levels of environment towards one offering no sensory stimuli whatsoever. 'Leveling up' in this way acts as both a motivator and a reminder for users to recognize and be mindful of the hindrance [19, 20].

\section{B. Ill Will}

Feeling unappreciated, frustrated at work, or jealous of ones colleagues and/or friends may foster such resentment and bitterness that one's thoughts are monopolized by negativity. Tailored environments can offer respite from such nagging distractions. Encouraging the meditative practice of 'Metta', (loving kindness), may offer profound adaptation away from such unfavorable thought processes.

Metta comes with its own 'leveling up' approach built in. The practice includes repeating a series of phrases (mantras) wishing a target individual health, happiness, and general well-being [21, 22]. One might begin by focusing mantras upon oneself. The next step may include those dearest to the practitioner. The ultimate goal is to progress through 'levels' of target individuals until such time that it is possible to genuinely wish the same well-being to those most likely to provoke negative thoughts. [22]. Gamification grants the possibility of directly visualizing a target. A practitioner may upload pictures directly into their simulation which become avatars ready for selection. By ordering the avatars created a practitioner is able to work through each in a way analogous to a gamer defeating each 'mini boss' on their way to the toughest 'final boss' at the end of the game.

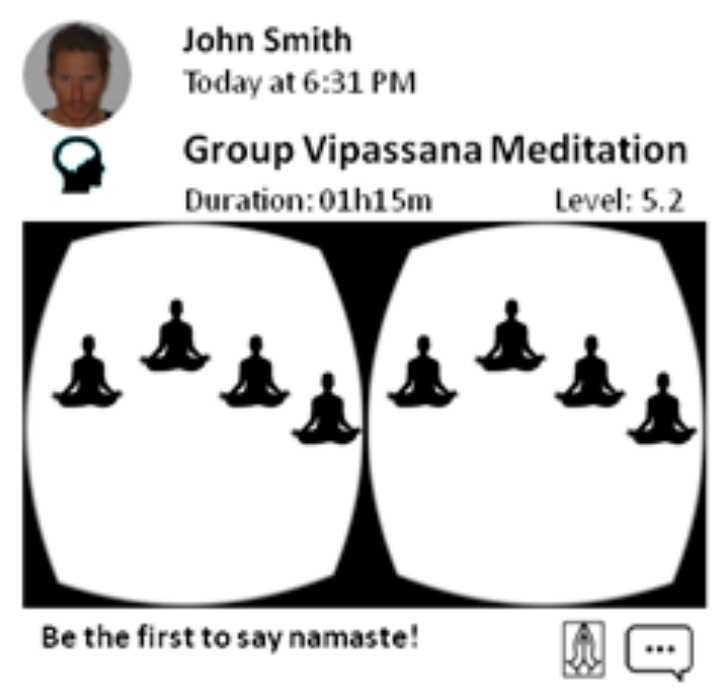

Fig 1. User's meditation practices appear on the activity feeds of friends and followers. 


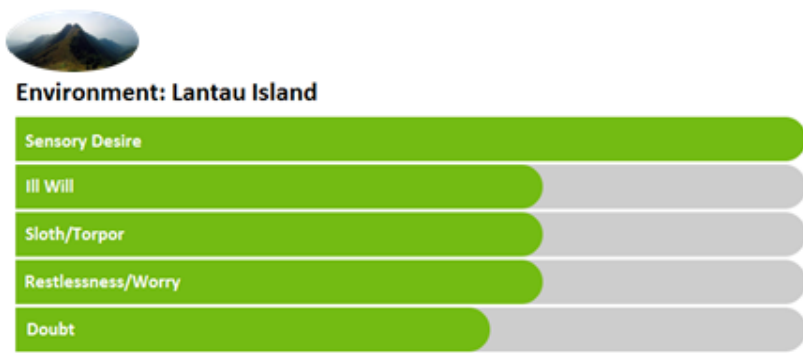

Fig 2. Meditation virtual environment selection depends on a user's current ability/skill level.

\section{Sloth \& Torpor}

The relaxing quality of mindfulness meditation naturally possesses the potential to over relax to the point where focus on the breath or alternative focal point is lost. Falling asleep, or becoming mentally sluggish to the point that the practice is no longer actual meditation, hinders practice [23]. Virtual meditation environments shall be designed in a way to address the problem head on for those new to meditation, or particularly susceptible to the hindrance. Once again, we can employ the concept of 'leveling up' to encourage mindfulness of the given hindrance while simultaneously increasing the motivation to progress beyond the hindrance's limitations.

Interactive technologies allow us to directly address this phenomenon. A head mounted display (HMD) and heart rate monitor (HRM) provide continuous data which, when cross-referenced, can offer a reliably probabilistic assessment of a user's current state of consciousness. Should an application detect a high likelihood of a user falling into an unhelpful state it can alert the user and update the environment in order to decrease the chance of recurrence.

\section{Restlessness \& Worry}

Meditation practice plagued by restlessness and worry may not only waste the time of the practitioner, but increase their concerns. Awareness of the inability to calm the mind is analogous to a sufferer of insomnia laying in bed thinking about the fact that they cannot sleep and how tired they feel. Wrestling with the resulting frustration only adds to the problem and decreases the chance of meaningful practice.

Gamification allows us to tailor simulations to help reduce such hindrances. Rather than entering the meditation environment directly a user may choose a 'virtual journey' to get to their meditation environment. When considering the deserted beach environment we may simulate a gentle sailboat ride in which the user is given the opportunity to escape their worries before starting their practice. Motion data from a head mounted display enables similar predictive models of a users restlessness. If detected we may provide alerts, guidance, and options in order to counter the problem.

\section{E. Doubt}

A lack of conviction that the meditation practice is benefiting a practitioner is common [24]. Much patience is often necessary in order to feel a palpable sense that meditation is worthwhile in general, and that the user feels themselves capable of accessing such benefits. The 'leveling up' we've described allows users a direct insight into both areas of concern.

The provision of statistics detailing a user's meditation experiences encourages self recognition and acceptance of subtle achievements and changes. Graphical visualizations to illustrate progress through levels, improvements in heart and breathing rates, and duration and regularity of practice, all add to a user's self and practice belief. Such statistical representations add much to a user's experience in both Strava and Zwift. Such feedback come in many guises. From simple encouragments regarding improvements in trend, to very detailed analyses for those more data driven. Providing a social network type platform akin to that of Strava shall help further. Support and encouragement from the community helps to reassure. An ability to follow others' meditation activities shall increase the overall acceptance and trust in the practice.

\section{Immersive Meditation PRACtice Using Virtual REALITY}

Providing everything described in virtual reality offers the greatest potential. Virtual reality remains novel to most users and so inherently reduces boredom. The ability to provide bespoke environments to users increases the efficacy of practice by uniformly combating the five hindrances described. Additional benefits come from VR's potential to evoke immersion and the resulting psychological feeling of presence.

We may increase the sense of presence by allowing a user to completely tailor their meditation environment. Choices of location and the ability to modify shall increase a user's ownership of their experience and in turn increase regularity of use. Modification shall increase presence by providing the brain with environments most accepting to it. A user's ability to switch and modify the avatar which they inhabit shall further increase presence. Selecting the right body type and clothing shall increase the overall sensory hijack [25].

The 'Proteus Effect' is an established phenomenon in VR [26]. We see behavior changes in a user both during and after stepping into an avatar dissimilar to 
themselves [27]. While this tactic may slightly reduce presence we may be able to harness the effect for good. Particularly hard cases who struggle to effectively meditate may benefit from stepping into the avatar of a meditation guru. Avatars of this kind may be equally effective when sitting opposite a user. By meticulously guiding the practice while exuding the highest levels of calm, enlightened, reassuring presence, we may again, see increased efficacy for even those hardest cases.

'Flow' is a state of mindfulness in which one is fully engrossed in an activity, characterized by heightened focus, loss of time awareness, and enjoyment in the activity [28]. VR has been shown to increase the likelihood of entering such a state [29]. Our ability to offer bespoke environments and simulations further increases this likelihood by offering user's a potentially optimum balance of challenge and skill [30]

\section{Applications in Mental Health AND PRisoner REHABILITATION}

We see concerning upward trends in the prevalence of a number of mental health disorder [31, 32, 33]. Novel therapeutic applications employing VR [34, 25] have shown promising results. Vipassana meditation shows similar promise as a therapeutic solution for alcohol and substance abuse [35], anxiety and depression [36, 37, 38], and stress [39].

While such trends are of course alarming in the general population, the issue is magnified within prison populations. Prisoners are far more likely to have psychosis and major depression. The prevalence of antisocial personality disorder is an order of magnitude higher than that of those not incarcerated [40].

Prison populations are increasingly being exposed to mindfulness meditation in order to rehabilitate [9, 41] and reduce stress, aggression, and substance abuse [42, 43, 44]. Virtual reality based practice may further increase participation among prisoners. The ability to virtually escape the confines of the prison for thirty minutes per week in order to sit on a virtual beach and meditate seems highly attractive.

The health benefits from mindfulness meditation for those with mental health disorders, prisoners, or the population in general is difficult to question. The flexibility of simulation afforded by VR enables bespoke scenarios designed specifically to address a given individuals needs. This may be chosen to directly counter one of the five hindrances described. Equally, our ability to tailor the experience/environment enables us to target specific concerns within a patient or prisoner. Some mental health conditions may be more suited to solo practice while others may be helped using group sessions. Similar suitability shall exist regarding the level or realness of an avatar, from cartoon-like through a pre-recorded, pre-rendered real-world
Vipassana teacher such as Matthieu Ricard or Joseph Goldstein [45].

The ability to absolutely control a virtual environment allows very high levels of replication [46]. As such, virtual meditation simulations which proved most effective would continue to be utilized for individual prisoners as well as future prisoners with similar psychiatric diagnoses. Such precise replication affords additional benefits in terms of assessing the overall efficacy of a given VR-meditation intervention.

Greater assessment of individual prisoners using such interventions is provided through the hardwear inherent to many high end VR systems. The ability to accurately track body movement, head movement, and gaze offers much in terms of user engagement. Such data offer additional utility in the potential diagnosis of a number of neurodevelopmental and motor system disorders[47, 48].

\section{Discussion}

The benefits of mindfulness meditation are clear. We illustrate how gamification in general, and specifically using VR, mitigates the obstacles inherent to the practice.

We describe how the best practices employed by Strava and Zwift are directly applicable to meditation practice. Developers at Zwift showcased a beta version of VR Zwift in January 2016. A commercially available version remains to be seen. Until the hardware problem is solved so that HMDs don't die due to user sweat we are unlikely to see a change. Meditation does not suffer the same problem.

While there already exist VR meditation apps, thus far they lack much of the functionality, and resulting utility described. This missed opportunity is likely due to developers prioritizing being first to the table. We encourage the evolution of meditation applications by focusing greater attention on the five hindrances while always mindful of the benefits of utilizing gamification techniques.

Mindfulness meditation is steeped in tradition. We intend no disrespect and laud the practice as a force for greater mental health. By highlighting the similarities with other healthy activities we hope to demystify and break the potential taboo of gamifying the practice. By making the practice more fun, attractive, and dare we say, addictive, for new practitioners we look to enhance new user experience and motivate more to take up, and stick with the practice.

We posit that the path to enlightenment must not necessarily avoid advances in modern technological. As with many beneficial practices, be they healthy eating, exercise, or abstaining from destructive or addictive behaviors, those first tentative steps are often the most 
difficult. By utilizing gamification and virtual reality into meditation practice we hope to encourage many to begin the path to better mental health. Once far enough along the path, such crutches are unlikely to be required. For those looking to explore a given practice more deeply the shedding of the HMD can represent a significant moment in their development [49].

\section{ACKNOWLEDGMENT}

This work has benefited from discussions with Matthew Harrison. We are also grateful for the valuable comments from the anonymous reviewers.

\section{REFERENCES}

[1] Holger Cramer, Helen Hall, Matthew Leach, Jane Frawley, Yan Zhang, et al, "Prevalence, patterns, and predictors of meditation use among us adults: a nationally representative survey", Scientific reports, 6, 2016.

[2] Adam Burke, Chun Nok Lam, Barbara Stussman, and Hui Yang, "Prevalence and patterns of use of mantra, mindfulness and spiritual meditation among adults...", BMC complementary and alternative medicine, 17(1), 2017.

[3] Philip A Chan and Terry Rabinowitz, "A cross-sectional analysis of video games and adhd symptoms in adolescents", Annals of General Psychiatry, 5(1):16, 2006.

[4] Kimberly Young, "Understanding online gaming addiction and treatment issues for adolescents", The American journal of family therapy, 37(5):355-372, 2009.

[5] Strava. www.strava.com/. (Accessed on 02/13/2018).

[6] Zwift. https://zwift.com/. (Accessed on 02/13/2018).

[7] Paul Grossman, Ludger Niemann, Stefan Schmidt, and Harald Walach, "Mindfulness-based stress reduction and health benefits: A meta-analysis", Journal of psychosomatic research, 57(1):35-43, 2004.

[8] Alberto Chiesa and Alessandro Serretti, "A systematic review of neurobiological and clinical features of mindfulness meditations", Psychological medicine, 40(8), 2010.

[9] Alberto Chiesa, "Vipassana meditation: systematic review of current evidence", The Journal of Alternative and Complementary Medicine, 16(1):37-46, 2010.

[10] XIV, Dalai Lama, et al., "In the buddha's words: An anthology of discourses from the pali canon", Simon and Schuster, 2005.

[11] David McMahan, "The making of buddhist modernism", Oxford University Press, 2008.

[12] Richard Gilpin, "The use of theravada buddhist practices and perspectives in mindfulness-based cognitive therapy", Contemporary Buddhism, 9(2):227-251, 2008.

[13] Andrew Olendzki, "Mindfulness and meditation", in Clinical handbook of mindfulness, pages 37-44. Springer, 2009.

[14] Henepola Gunaratana, "The path of serenity and insight: an explanation of the Buddhist jhanas", Motilal Banarsidass Publ., 1985.

[15] Jeroen Stragier, "What can we learn from strava?", Victoris Ghent University, http://www.victoris.be/what-can-we-learnfrom-strava/ (Accessed on 09/13/2018).

[16] Simon Schneider, Armin Senoner, and Danielle Gratch, "What Airbnb and Strava Know About Building Emotional Connections with Customers", Harvard Business Review, https://hbr.org/2018/05/what-airbnb-and-strava-know-aboutbuilding-emotional-connections-with-customers (Accessed on 09/13/2018)

[17] Elizabeth C Hirschman and Morris B Holbrook, "Hedonic consumption: emerging concepts, methods and propositions", The Journal of Marketing, pages 92-101, 1982.

[18] Kent C Berridge, Chao-Yi Ho, Jocelyn M Richard, and Alexandra G DiFeliceantonio, "The tempted brain eats: pleasure and desire circuits in obesity and eating disorders", Brain research, 1350:43-64, 2010.

[19] Mark T Greenberg and Joy L Mitra, "From mindfulness to right mindfulness: The intersection of awareness and ethics", Mindfulness, 6(1):74-78, 2015.

[20] Joseph Goldstein, "The experience of insight", Shambhala Publications, 2017.

[21] Emma M Seppala, Cendri A Hutcherson, Dong TH Nguyen, James R Doty, and James J Gross, "Loving-kindness meditation: a tool to improve healthcare provider compassion, resilience, and patient care", Journal of Compassionate Health Care, 1(1):5, 2014

[22] Greg Feldman, Jeff Greeson, and Joanna Senville, "Differential effects of mindful breathing, progressive muscle relaxation, and loving-kindness meditation on decentering and negative reactions to repetitive thoughts", Behaviour research and therapy, 48(10), 2010

[23] Robert R Pagano, Richard M Rose, Robert M Stivers, and Stephen Warrenburg, "Sleep during transcendental meditation", Science, 191(4224), 1976.

[24] Joseph Goldstein and Jack Kornfield, "Seeking the heart of wisdom", Shambhala Publications, 2001.

[25] Giuseppe Riva, "Virtual reality in psychotherapy", Cyberpsychology \& behavior, 8(3):220-230, 2005.

[26] Nick Yee and Jeremy Bailenson, "The proteus effect: The effect of transformed self-representation on behavior", Human communication research, 33(3):271-290, 2007.

[27] Nick Yee, Jeremy N Bailenson, and Nicolas Ducheneaut, "The proteus effect: Implications of transformed digital selfrepresentation on online and offline behavior", Communication Research, 36(2):285-312, 2009.

[28] Mihaly Csikszentmihalyi, "The future of flow", 1988.

[29] Li-Keng Cheng, Ming-Hua Chieng, and Wei-Hua Chieng, "Measuring virtual experience in a $3 \mathrm{~d}$ vr interactive simulator environment: a structural equation modeling approach", Virtual Reality, 18(3):173-188, 2014.

[30] Mihaly Csikszentmihalyi, "Literacy and intrinsic motivation", Daedalus, pages 115-140, 1990.

[31] AH Weinberger, M Gbedemah, AM Martinez, D Nash, et al., "Trends in depression prevalence in the usa from 2005 to 2015: widening disparities in vulnerable groups", Psychological medicine, 2017.

[32] National Institutes of Health et al., "Ptsd: A growing epidemic", NIH Medline Plus, 4(1):10-4, 2009.

[33] MD Laxmaiah Manchikanti, II Standiford Helm, and Jeffrey W Janata MA, "Opioid epidemic in the united states", Pain physician, 15, 2012.

[34] Lynsey Gregg and Nicholas Tarrier, "Virtual reality in mental health", Social psychiatry and psychiatric epidemiology, 42(5):343-354, 2007.

[35] G Alan Marlatt, Katie Witkiewitz, Tiara M Dillworth, Sarah W Bowen, George A Parks, et al., "Vipassana meditation as a treatment for alcohol and drug use disorders", Mindfulness and acceptance, 2004.

[36] Stuart J Eisendrath, Kevin Delucchi, Robin Bitner, Paul Fenimore, et al., "Mindfulness-based cognitive therapy for treatment-resistant depression", Psychotherapy and Psychosomatics, 77(5), 2008.

[37] Sameet Kumar, Greg Feldman, and Adele Hayes, "Changes in mindfulness and emotion regulation in an exposure-based cognitive therapy for depression", Cognitive Therapy and Research, 32(6):734, 2008.

[38] Jonathan R Krygier, James AJ Heathers, Sara Shahrestani, Maree Abbott, James J Gross, and Andrew H Kemp, "Mindfulness meditation, well-being, and heart rate variability", International Journal of Psychophysiology, 89(3), 2013.

[39] Roberta A Szekeres and Eleanor H Wertheim, "Evaluation of vipassana meditation course effects on subjective stress, wellbeing, self-kindness and mindfulness in a community sample: 
Post-course and 6-month outcomes", Stress and Health, 31(5):373-381, 2015.

[40] Seena Fazel and John Danesh, "Serious mental disorder in 23000 prisoners: a systematic review of 62 surveys", The lancet 359, no. 9306 (2002): 545-550.

[41] Sam Himelstein, Arthur Hastings, et al., "Mindfulness training for self-regulation and stress with incarcerated youth: A pilot study", Probation Journal, 59(2), 2012.

[42] Sarah Bowen, Katie Witkiewitz, et al., "Mindfulness meditation and substance use in an incarcerated population", Psychology of addictive behaviors, 20(3):343, 2006.

[43] Marlene Samuelson, James Carmody, Jon Kabat-Zinn, and Michael A Bratt, "Mindfulness-based stress reduction in massachusetts correctional facilities", The Prison Journal, 87(2):254-268, 2007.

[44] Atefeh Milani, Zahra Nikmanesh, and Ali Farnam, "Effectiveness of mindfulness-based cognitive therapy (mbct) in reducing aggression of individuals at the juvenile correction and rehabilitation center", International journal of high risk behaviors \& addiction, 2(3), 2013

[45] U Volpe, B Dell'Osso, A Fiorillo, D Mucic, and E Aboujaoude, "Internet-related psychopathology: clinical phenotypes and perspectives in an evolving field", Official Journal of the Italian Society of Psychopathology Organo Ufficiale della Societa Italiana di Psicopatologia, 46:406- 414, 1989.

[46] Bobbie Ticknor and Sherry Tillinghast, "Virtual reality and the criminal justice system: new possibilities for research, training, and rehabilitation", Journal For Virtual Worlds Research 4, no. 2 (2011).

[47] Daniel S. Pine, Joseph Grun, Eleanor A. Maguire, Neil Burgess, Eric Zarahn, et al., "Neurodevelopmental aspects of spatial navigation: a virtual reality fMRI study", Neuroimage 15 , no. 2 (2002): 396-406.

[48] Stephen J. Brown, "Method for diagnosis and treatment of psychological and emotional conditions using a microprocessorbased virtual reality simulator", U.S. Patent 6,186,145, issued February 13, 2001.

[49] Daniel P Brown, "Pointing out the great way: The stages of meditation in the Mahamudra tradition", Simon and Schuster, 2006. 